\title{
The Simplicity of Complex Systems: The Inquiry into the Nature of Life, Mind, and Death Phenomena (Essay)
}

\author{
Mark Iosim \\ Independent Researcher, USA \\ *Corresponding Author: markiosim@gmail.com
}

Copyright $(\mathcal{C} 2016$ by authors, all rights reserved. Authors agree that this article remains permanently open access under the terms of the Creative Commons Attribution License 4.0 International License

\begin{abstract}
The main goal of this paper is the search for the explanation of life phenomena. These days it is common to employ the concept of emergence as a gateway for an explanation of complex system phenomena like mind and life. However, after almost a century of development, emergentism has not demonstrated that it is a viable alternative to reductionism. In this paper emergence is viewed as an illusion caused by hidden properties of parts observable only during interactions in the system; the system acts as "litmus test" or a "magnifying glass" revealing the parts' properties not observable otherwise. This methodology, when pushed to its limits, leads to the radical conclusion that life phenomena may not be explained within the boundary of existing physical paradigms. Instead, it is argued that panpsychism, which recognizes mental capacity as a fundamental property of matter, is superior to physicalism in explaining not only Life and Mind, but also the notoriously controversial topics of quantum mechanics. There is a high price for accepting this view, because it requires the rejection of fundamental scientific paradigms. However, the reward for the transition to this new paradigm justifies the cost, because the new approach promises to eliminate "weirdness" from the interpretation of quantum mechanics, unite physical and life sciences, and bridge science with spiritual experience. In contemporary science, panpsychism experiences broad unacceptance and is seen as merely a vestige of primitive pre-scientific beliefs. To be taken seriously, panpsychism needs to demonstrate its superiority in the interpretation of natural phenomena. Several examples demonstrating the power of the new paradigm in solving the most challenging problems faced by science are presented in this paper. The examples cover a wide range of scientific inquiries such as interpretation of quantum mechanics, the theory of consciousness and intelligence, emergence of life.
\end{abstract}

Keywords Panpsychism, Emergence, Reductionism, Mind, Intelligence, Emergence of Life, Death

\section{Introduction: From Emergentism to Holistic-Reductionism}

There are two main rival approaches to scientific inquiry: reductionism and holism. According to reductionism, all properties of a system could be derived from studying its parts and their interactions, using fundamental physics and chemistry. Reductionism is the dominant paradigm in sciences. However, in spite of spectacular successes, reductionism is having difficulty encompassing the most intriguing problems of our time: explaining life and mind phenomena. As critics pointed out, reductionism is a limited methodological tool that over-focuses on isolated parts and therefore it cannot be applied to complex systems [49]. From this standpoint, reductionism is contended with holistic views, which state that the essential property of a system, as a whole, cannot be explained using properties of its elements. The system's essential properties appear emergent.

The emergence paradigm is commonly employed as a gateway in an explanation of complex system phenomena like mind and life. However, in spite of its attraction the emergentist strategy is regarded with suspicion by many contemporary philosophers [52]. After almost a century of development, emergentism has not demonstrated that it is a viable alternative to reductionism either.

Traditionally, emergence is associated with dynamic systems whose behavior cannot be predicted from knowledge about parts studied in isolation. This is the biggest confusion in emergentism, caused by failing to recognize that isolated parts, in the absence of interaction, will not exhibit any properties at all. For example, we know that an electron possesses an electrical charge, but we cannot observe this property unless electron interacts with another charged particle. We do not declare that the charge of an electron emerges during interaction. Instead we agree that the electron always posses the charge (whether we observe it or not) and reveals this property only during interactions.

The typical rhetorical argument in favor of emergence is the taste of sugar, that could not be found in the carbon, hydrogen, and oxygen atoms that constitute components of 
sugar molecules [48]. This example, limits the whole to sugar only while the another crucial element of the system is missing; someone who tastes the sugar and constitutes the sugar taste property. In the majority of emergence examples there is an exclusion of the invisible someone who designs, tests, or observes. Without this someone, the system property, like the taste of sugar, or "wetness" of water would not exist at all. Omitting the creator or user of the system is the far most common mistake in emergentism. For example, a complex computer is built from simple semiconductor components and it seems that the "computational intelligence" of the computer is a new emerging phenomenon, because it cannot be found in its parts. However, the "intelligence" of the computer is also due to human intelligence, which is not seen while we are observing the computer. Therefore, human intelligence is also one of the system's causal powers and his or her properties determine the complexity of the semiconductor components, the complex wiring of the logic diagram, and sophisticated algorithms. In other words, there are no emerging properties in this example and the properties of a computer could be reduced to the properties of its constituents, including the creators of this computer.

Further examination of the evidences supporting emergence phenomena led me to the conclusion that emergence is a misperception caused by "hidden properties" of parts observable only during interactions in the system giving an impression of emerging properties; a system acts as "litmus test" or a "magnifying glass" that just reveals the parts' properties not observable otherwise. This methodology, named "Holistic Reductionism," could be summarized as follows:

1. The perception of emergence is due to interactions in a system that reveals the parts' hidden properties not observable in isolation.

2. The nature of complex things is always reducible to the nature of the sum of underlying constituents and their causes and therefore a whole is always equal to the sum of its parts, where the sum is any mathematical or logical procedure that evaluates a resultant of multiple causes.

3. If the system exhibits properties that cannot be reduced to the underlying mechanisms, they may be caused by the parts' hidden properties, so additional efforts to discover them are necessary.

\section{Hidden Properties of Macromolecules}

Let us use the methodology of holistic-reductionism to analyze the behavior of the simplest unicellular organisms the humble paramecium.

Regardless of its simplicity, the humble paramecium behaves in a very sophisticated manner. It swims with its numerous tiny hair-like legs (cilia) darting in the direction of bacterial food, or retreating at prospect of danger, ready to swim off in another direction. This unicellular organism can also negotiate obstructions by swimming around them and demonstrates other complex behavior.

In general, the most basic properties of a unicellular organism can be reduced or at least associated with the properties of its parts. For instance, a cell's ability to perceive the external information are associated with the membrane proteins sensitive to the external factors; the ability of locomotion is due to the property of the flagellum-like structures. Yet, the cell's ability to process information and act accordingly appears irreducible. The attempts to explain the cell's complex behavior in terms of underlying mechanisms have continued for a long time, but without noticeable progress.

According to methodology of holistic-reductionism, if a system exhibits properties that cannot be reduced to the underlying mechanisms, the problem could be caused by the parts' hidden properties. Following this principle, we will attempt to search for the hidden properties of sub-cellular constituents that may be responsible for the complex behavior of unicellular organisms.

In the entire realm of intracellular organization, no class of molecules can compete with proteins. Many proteins are enzymes that catalyze biochemical reactions and are vital to every process within cells. Their functions and mechanisms have been studied in great detail, but still are not fully understood. In most cases, enzyme mechanisms are explained in terms of selective binding with molecular substrates, thus forcing them into specific biochemical reactions. The three-dimensional shape of a protein is vital for this function. The mechanism by which proteins undergo folding into their native three-dimensional shape is one of the most studied but is still not fully understood. In general, it follows the minimum of free energy, however if a protein were to fold by randomly sampling of all possible conformations, it would take about 1010 years to finish folding. Cyrus Levinthal, who first noticed this paradox in 1969 , proposed that a random conformational search does not occur in folding, and therefore the protein must fold by a directed process. Indeed, specialized proteins, called chaperones, whose function is to aid in the folding of other proteins, were later found. However, protein self-folding is also observed, so the protein folding paradox still has no satisfactory explanation [47]. It is clear that the polypeptide chains do not try all possible 1060 conformations, but somehow find the appropriate one.

Another example of the sophisticated behavior of enzyme molecules is the DNA polymerase that control DNA synthesis. First, this enzyme catalyzes the synthesis of DNA, and then checks its correctness; if an incorrect base pair is recognized, the same DNA polymerase enzyme reverses its direction, eliminates the incorrect base pair, and continues replication. This two-step process is called 'proof-reading' and results in average error rates of less than 1 error in a billion nucleotides [44]. The fact that a molecule can perform such complex functions could create the impression that it has a "mind" of its own, rather than following the spontaneous, free-energy reduction driven processes. It is 
important to keep in mind that the term spontaneous doesn't explain the complex molecular behavior and instead just conceals a gap between physical forces acting in a linear manner, and the complex behavior of macromolecules. It is why they are often described in the metaphysical terms of a goal-directed, free energy reduction processes.

Stephen Wolfram, in his book, A New Kind of Science [16], proposes the Principle of Computational Equivalence according to which all processes occurring spontaneously in nature can be viewed as computations following the basic laws of nature.

Let us spinoff from this principle and assume for a moment that proteins molecules indeed possess some sort of "molecular computational ability." Thanks to this ability, protein molecules could "figure out" how to undergo the folding process, control the biosyntheses of other macromolecules, collectively manage the cell's regulatory mechanisms, etc. In general, the complex regulatory and behavior mechanisms of living cells could be reduced to "molecular computational ability."

If we accept that molecules may perform complex "computations" and if we adhere to the method of holistic-reductionism, we will be forced to admit, at least as a possibility, that the causation of "molecular computational ability" could be hidden even deeper, on the level of atoms and subatomic structures.

This inference was as unexpected to me then as it is probably unacceptable for the majority of readers of this essay now. Even though this idea is not supported by today's science, I am not aware of impossibility of this proposition. Therefore, admitting that this is a highly speculative inquiry, we should be permitted to continue this analysis.

\section{Panpsychism}

If we follow the inference from the previous chapter, we have to admit, at least as a possibility, that atoms and even elementary particles may have some sort of computational abilities. By making this assumption, we are stepping on the slippery slope of panpsychism, which recognizes a mental capacity as fundamental property of matter. Panpsychism is not a formal theory of mind and does not necessarily attempt to define mind, nor does it necessarily explain how mind relates to the objects that possess it. Therefore, panpsychism is more of an overarching concept, a kind of meta-theory that views mind as fundamental to the nature of existence $[1,2]$. Panpsychism has a long and noble tradition from Greek to western philosophy. Many distinguished scientists and philosophers of the mid-20th century $[3,4,5,6]$ and present days $[1,7,8,9]$ were inspired by panpsychism. However, in contemporary sciences panpsychism experiences broad unacceptance and is seen as merely a vestige of primitive pre-scientific beliefs. Having enough trouble being taken seriously for its substance, the name itself invokes mythology.

Perhaps the most obvious problem with panpsychism is lack of evidence that the physical world possess any mental characteristics [7]. Nevertheless, it could be argued that all physical phenomena that currently described in terms of the external forces acting on the inanimate world instead could be interpreted in terms of goal-directed mental processes occurring inside elementary particles. For example, an electron could be viewed not as an entity that blindly follows external electrical forces, but a free self-propelled entity that is attracted and therefore actively searching for positively charged particles. Obviously, this is not what we learned in school about physical forces.

Historically, the concept of force, taken originally in analogy to human will power, spiritual influence, or muscular effort, became projected into inanimate objects. In elementary or introductory courses in physics, a force is still interpreted in the traditional animistic manner as a tendency or striving or attraction. In quantum chromodynamics though, the standard model of force is replaced by the ontologically less demanding concept of interaction, which manifests the exchange of virtual particles that mediate this interaction. Clearly, what one calls the fundamental forces of nature are no longer forces in the traditional sense. Modern particle physics seems to support the thesis that the concept of forces has reached the end of its life cycle even though the term force continues to be part of our scientific vocabulary [10].

If an external force, as physical reality, does not exist, the only source of motion is in the particles themselves, so they could be viewed as independent goal-directed entities and their interactions as an informational exchange. Electrons, for example, while interacting with positively charged particles exchange information (some mediating elements) and if the exchange is beneficial for both interactive particles (lower their free energy) they are attracted to each other this could be the essence of the physical force. The stronger the attraction between particles the more obstacles they are 'willing' to overcome on their way toward each other - this could be the essence of physical energy. We may also introduce memory as the fundamental property of matter that is equivalent to inertia; a particle remembers the direction where the attraction/repulsion came from and continues moving in the same direction even after the attraction/repulsion is gone. This way, the memory of a particle is proportional to its inertial mass. For example, the size of memory of neutrons or protons is thousands of times larger than that of electrons.

Similarly, all known physical phenomena may be expressed in terms of particle's freedom of choice, goal, ability to make decision, etc. It means that if matter looses its 'mind-like' properties, the physical properties would disappear too.

From the perspective of the majority, panpsychism seems to complicate our views on the relatively simple physical processes and in general is a wacky approach to explaining the natural world. For those who agree with this characterization of panpsychism, I suggest to recall the orthodox quantum interpretations, which are broadly 
accepted as plausible. For example, according to quantum mechanics, fundamental elementary particles exist in many places at once, and actually, they are not particles at all, but rather wave-like ripples in time-space, and by the way, we all exist in parallel universes. Contrary to this 'head-twisting' interpretation of the physical world, the new paradigm offers a realistic and coherent interpretation. For example, wave-particle duality of mater could be explained by a zig-zag trajectory of particle that scans the surrounding space in search for interaction. The notoriously controversial wave function collapse could be explained by particle ceases the zig-zag motion when it finds its target.

The idea that the quantum mechanics could be derived from the particle's zig-zag -like trajectory is not new. It was suggested by Richard Feynman, David Bohm, and Edward Nelson [51] but 'was dead on arrival' because according to the dominated views on physical reality it would require an external "wave like" force to cause this trajectory. In our quantum model, no such force is required. Of course, this subject required much more considerations, which cannot be presented here due to the limited format of this paper.

The views on the physical world as driven by mentality would bridge physical processes with mental and social processes of living organisms, and could explain the similarities between physical and social phenomena. There is an abundance of scientific observations exploring this similarity, regardless that physical processes are governed by supposedly simple mathematically defined forces, while human behavior is governed by a wealth of psychological complexity. For example, it was shown that the movement of people in crowds seems to obey the Maxwell-Boltzmann statistics of the kinetic theory of gases; in situations that enhance the crowd's density, a phenomenon similar to phase transition to a liquid-like state is observed [11]. By employing a model of "self-propelled particles" that obey simple rules of interaction, it is possible to model the dynamics of flocking birds, schooling fishes, the behavior of a human crowd, the complex phenomena of cultural segregation, the spread of crime, the formation and stability of international alliances, the transmission of cultural traits, and economic markets $[11,13]$.

Following the analogy between physical forces and processes in human society, political economist R.J. Rummel, introduced the notion of force fields into a social environment [14]:

\section{"...The socio-cultural field is a space-time continuum generated by individual needs. The activation of needs, their transformation from potentiality to actuality provides regions of energy in the field...Thus, the socio-cultural field is a force field..."}

The description of complex social processes using a physical model aims to provide the simplest possible description. The physical sciences are mostly successful in explaining our world using relatively simple mathematical formalism, thus it seems there is no room left for the theory that atoms and molecules hide intelligence. For intelligence, we would rather look into a telescope than into a microscope.

Let's put ourselves into the shoes of those whose intelligence may be ignored. Imagine that some extraterrestrial civilization is studying our planet from far away. Suppose that the extraterrestrials are millions of times bigger than us so for them we just a particle of dust. Because these extraterrestrials assume that all intelligent life forms in the universe should be similar to themselves, including in size, they do not expect to find any intelligence in the human particles. At the same time, extraterrestrial scientists are puzzled by the sophisticated infrastructures resulting from seemingly chaotic behavior of the human particles. After some examination of this phenomenon the extraterrestrials find the correlation between money and human behavior; all humans seem to be attracted to money with a force proportional to its quantity; the more money that is at stake, the more obstacles the human particles overcome in order to get them. This is very similar to the physical world; the motion/action of any physical object is proportional to the attracting force and inversely proportional to the magnitude of the obstruction. Following the principle of the simplest explanation, extraterrestrial scientists concluded that human's behavior is governed by the money force field that drives all human interactions.

However, exceptions to this rule are also observed; sometimes, some human particles act in violation to the money force field by ignoring them. Yet the extraterrestrial scientists defend their theory by pointing out that the quantum particles behave the same way. Their behavior also is not fully deterministic, and only the probability of the micro event is predicted by quantum mechanics. Using the money force theory, extraterrestrial scientists indeed were able to explain the majority of phenomena observed in human system. Therefore, it was concluded that no further study of the simple human particles is necessary.

Likewise, for a great majority of applied sciences, the atom is ground zero, the foundation of all other knowledge, and therefore there is no practical significance in exploration below this level. It is believed that if particle physicists will one day find a complete, profound, and satisfying explanation for the existence and properties of neutron, protons, and electrons, our knowledge of chemistry and biology will not benefit at all. Nevertheless, the foundation of our sciences may not be as sturdy as it appears. Despite the wide acceptance of quantum mechanics as the basis of physical scientific disciplines, the controversy around it that started more than eighty years ago is no mere historical footnote. It is still argued by some that quantum mechanics gave a useful but fundamentally incomplete account of the physical world and that certain vital areas of quantum mechanics are covered not by convincing computations, but by a transparent tissue of ideology. In the history of science the small group of mavericks, who have made a major contribution to quantum mechanics like Albert Einstein, Eugene Wigner, Erwin Schrödinger, David Bohm, and Paul Dirac expressed grave doubts that quantum theory gives us a 
true view on reality [15].

Advocating for panpsychism is an uphill battle, because it suggests a radically different worldview, challenging our imagination and existing paradigms at their roots. To be taken seriously, panpsychism need to demonstrate its superiority in the interpretation of natural phenomena. Several examples demonstrating the power of the new paradigm in solving the most challenging problems faced by modern sciences are presented below.

\section{Consciousnesses}

While considering the mind-like properties of matter we have to explain how they may relate to consciousness, that is, our subjective experience and awareness. The mystery of consciousness is certainly one of the most persistent problems in the philosophy of the mind and is the subject of much research in psychology, neuroscience, artificial intelligence, and is even discussed within framework of quantum mechanics.

Within the philosophy of mind, there are number of different approaches to this phenomenon, but the two major schools of thought are dualism and monism. According to dualism, there are two kinds of entities, matter, and mind. Religious and mystical teachings are prime examples of dualistic views. The central problem for dualists is how mind and matter relate to each other.

Monism is the position that mind and body are ontologically the same kinds of entities. For example, within the physicalist doctrine, consciousness refers to physical and chemical processes occurring in the brain.

The panpsychism advocated here holds monistic views on consciousness, because it presents mentality and physicality as two interpretations of the same reality; the universe is made of fundamental particles having consciousness and intelligence (although with a very impoverished degree and kind of content) that determine physical properties of matter.

While individual particles may contain a negligible level of conscience, the group that shares their conscience experience/information would form a collective/cumulative conscience. The collective consciousness could be defined as the total amount of information shared (circulated) among members of the system. This means that without sharing the information, the system cannot be considered conscious, regardless how conscious their members are.

To evaluate the system's collective consciousness it would be useful to introduce the factor of Relative Cumulative Consciousness (RCC) as follow:

$$
\mathrm{RCC}=\sum \mathrm{I}_{\text {shared }} / \sum \mathrm{I} \text { total } \times 100 \%
$$

where:

$\sum I$ total the total amount of conscious information available in the system's constituents (shared or not).

$\sum I_{\text {shared }}$ only shared conscious information.

For example, if all constituents in a system share $100 \%$ of their conscious information the systems' RCC is $100 \%$ and its cumulative consciousness reaches a maximum. A hypothetical example is a group in which the members are linked telepathically, thus having unrestricted access to each other's internal information. However, if there is no information sharing in the system, its RCC, and cumulative consciousness accordingly is zero, even though individual members may possess consciousness. These types of systems should be called aggregates. For example, noble gases could be an example of nearly zero conscious systems, because their molecules aren't involved in any attractive interaction (that constitutes information sharing). Actually, in this case it would be hard to call this gas a system, even if it is forced into some sort of boundary (like a bottle, tank, etc.) because the molecules do not comply with a system's definition as interdependent entities [53]. Rather, these types of entities should be called aggregates, (forced to stay together). Most non-living objects, a rock for example, could be viewed as an aggregate, because the physical integrity of a rock depends on the limited bonds between only the adjacent elements, which do not form network-like bonds. Some living and even social entities should be also called aggregates. For example, a population of inmates in prison that are forced into the boundary of the jail, or passengers on public transportation trapped within the boundary of a public vehicle do not form network-like bonds, practically don't share information, and therefore their RCC may not exceed that of a rock.
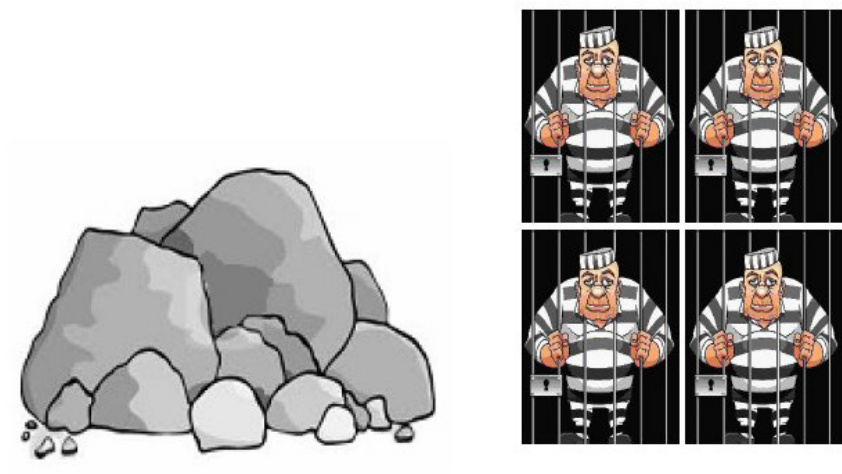

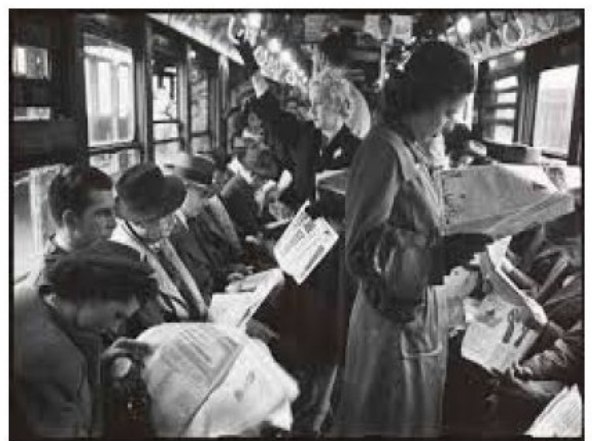

Figure 1. Aggregates 
Following the views on consciousness advocated here, the majority of conscious events, especially in non-living systems, are undetectable, because often we cannot observe informational exchange inside the system. However, we may examine the networking capabilities of the system as prerequisite for information sharing and based on this characteristic predict the possibility and degree of collective consciousness. Below we will examine the most common systems.

Let us start with an atom that consists of interacting protons, neutrons, and electrons.

The protons and neutrons interact by very strong nuclear forces while electrons interact with protons with much weaker electromagnetic forces. Based on the assumption that information exchange determines the strength of physical forces, it can be concluded that the information exchange within atomic nucleus is significantly higher than between protons and electrons. This means that the collective consciousness of an atom is mostly accumulated in the nuclei,. Taking in account the suggestion in the previous chapter that the inertial mass of a particle is the physical equivalent of its memory, the nucleus should be the major depository of an atom's conscious experience.

The next hierarchical level of matter is a molecule, in which two or more atoms share their outermost electrons resulting in interactions called chemical bonds. According to the Molecular Orbital Theory [17] electrons are not assigned to individual bonds between atoms, but spread over the entire molecule (as long as permitted by certain quantum rules) facilitating the sharing of conscious experiences among individual atoms and this way playing a key role in forming cumulative consciousness of the molecule. Obviously, the larger the molecule the higher its cumulative consciousness is. However, size by itself is not enough to achieve high cumulative consciousness. For example, in a large macromolecules existing in the form of a long, linear chain, electrons cannot provide information sharing between distant parts of the molecule. This is why polymer molecules of plastic, regardless of their size of several thousand repeat units, should have a relatively low RCC. Yet, if a long, linear macromolecule is folded into compact globular structure, it enables the interactions between its remote regions; this enhances the information exchange, and consequently results in a high RCC of this macromolecule. It may not be a coincidence that these types of macromolecules are prominent in biological structures.

An example of such molecule is DNA, which is made from the millions of repeating nucleotides. In the cell, the DNA molecule is supercoiled and folded in a way that makes interactions among distant parts of the DNA molecule possible, especially in the eukaryotic chromosome, where it is supercoiled around histone proteins forming fibers that are coiled upon themselves numerous times [18]. This tight packaging enables interactions among various parts of the DNA/protein conglomerate and as a result, chromosomes should have a very high RCC. Taking in account the enormous size of a chromosome, its total Cumulative
Consciousness could be the highest among other macromolecular structures of the cell. This makes me think that genomic DNA contains not only genetic information, in the form of DNA code, but also may store the epigenetic information in the form of conscious experiences inherited from the previous cell generations.

Another example of systems that may have a considerable degree of Relative Cumulative Consciousness are dissipative systems operating far from thermodynamic equilibrium. Examples are cyclones, hurricanes, Bénard cells, Belousov-Zhabotinsky reactions, etc. These systems exhibit a high degree of long-range correlations and act as a whole indicating network-like interactions that are prerequisite for the rise of collective consciousness. For example, in the Belousov-Zhabotinsky reaction, the characteristic of chemical oscillations indicate that molecules must have a way to "communicate" over a distance exceeding the normal range of interaction between molecules hundreds of millions of times [19]. Therefore, dissipative systems should have considerable information sharing within them, and therefore corresponding degree of Relative Cumulative Consciousness.

The next level of organization is a living cell. In context of thermodynamics, cells exist in a state far from equilibrium and many of their mechanisms are examples of self-organized dissipative systems. A cell maintains itself through the constant exchange of products of metabolic reactions migrating within the cell, often over long distances. This could be viewed as an informational exchange that complies with a prerequisite for collective consciousness.

The most visible role in controlling metabolic mechanisms and communication within the cell belongs to protein molecules. Using the extensive network of endoplasmic reticulum as "public transportation," proteins can travel throughout the cell, often being marked with short signal sequences of amino acids that function like a postal code for the target destination (Campbell [2002], p. 320). Proteins may also exchange information using intra-protein communication by which proteins interact by modifying their structural microstates [20]. A vast number of these microstates, like "molecular sign language," could convey a complex message, and this way may enable the sharing of a complex conscious experience. Taking in account the sophistication of controls provided by proteins and their role in the information sharing within cell, collectively, proteins should play a key role in the cell's intelligent abilities and cumulative consciousness.

Moving from a cell to an organism, we arrived at the main battlefield where various competing philosophical and scientific theories are trying to solve the mind-body problem. The views on consciousness advocated here simplifies this problem, because it is expected that the cells of our body already have some degree of consciousness, so what is left is to explain how the inferior consciousness of cells is transformed into the superior collective consciousness of the organism, a human for example. We have to just look for the place in our body that complies with the prerequisite for 
collective consciousness, the place where the conditions for information exchange among cells are the most favorable.

If we knew nothing about our body, but only its detailed microanatomy, we should be able to conclude that the most conscious part of our body is the brain, because its cells form the most prevalent communication network. By further examining neuron connections in the brain, we would find that the highest degree of networking is achieved within cerebral cortex, which has a vast majority (as high as 99\%) of connections within itself rather than with subcortical areas [21]. Therefore, the cerebral cortex should play a dominant role in representing our consciousness. That is in agreement with traditional views on the role of cerebral cortex in human consciousness [22, 23].

The evolution of human communication, from speech, writing, printing, radio, TV and finally internet and social media, enhanced the sharing of our everyday experience, emotion, knowledge, thoughts etc. Our awareness of the world is no longer limited to our senses; we learn from each other's experiences, and so begin to accumulate a collective body of knowledge about the world that links us into one worldwide community. In The Global Brain, Peter Russell argues that humanity is on its evolutionary path to a "global brain" of collective consciousness [24]. It is hard not to agree with Russell's assessment about the evolution of human organization. Our society still has a long way to go until we reach the RCC of our brain or even the social organization of ants and bees, which seems are way ahead of us.

\section{Redefining Intelligence}

Despite the variety of concepts of intelligence, practically all of them define intelligence in terms of various human-like mental abilities, but there is no agreement which trait is more important in defining intelligence. It seems that the most promising is the theory of Multiple Intelligences developed by Howard Gardner by which human intelligence entails a set of specific skills and abilities [25]. Gardner argues that intelligence, as it is traditionally defined, does not sufficiently encompass the wide variety of abilities humans display. For example, a child who masters multiplication easily is not necessarily more intelligent overall than a child who struggles to do so. The second child may best learn through a different approach, or may even be looking through the multiplication learning process at a fundamentally deeper level that hides a potentially higher mathematical intelligence than in the one who memorizes the concept easily. Some who cannot do well in mathematics could be stronger in another kind of intelligence and may excel in a field outside of mathematics.

Using the theory of Multiple Intelligences and combining it with the Stephen Wolfram's Principle of Computational Equivalence that "all processes occurring spontaneously in Nature can be viewed as computations" [26], we can build the new general theory of intelligence that incorporates not only human intelligent abilities, but intelligent abilities for any living or non-living system.

The foundation of this theory of intelligence is the notion that any process occurring in living or nonliving nature can be viewed as a goal-directed problem solving process to achieve maximum entropy, or adaptation, etc. For example, the trajectory of a thrown stone, the self-assembly of molecules, snowflake formation, or any adaptive behavior of living organisms, all could be viewed as problem solving processes. This way the synthesis between the theory of Multiple Intelligences and Principle of Computational Equivalence could be formulated as follows:

Intelligence is a collection of the specific problem solving (intelligent) abilities, where, solving a problem is any spontaneous or goal-directed adaptive behavior of any living, non-living or artificial system.

According to this theory, we cannot measure intelligence as a whole, but specific abilities only. Each individual ability could be measured in the process of interaction with a specific problem or class of problems.

\section{The value of a specific intelligent ability to solve a specific problem or specific class of problems (within specific timeframe) could be determined as the probability $\boldsymbol{p}$ of a correct answer.}

The value of specific intelligent ability (lets call it a Grade) could be ranged from 0 to 1 . For example, if an individual has been solving specific types of problems with $50 \%$ success rates, this person's intelligence, relative to this type of the problems, is 0.5 grades.

\section{Who is more intelligent?}

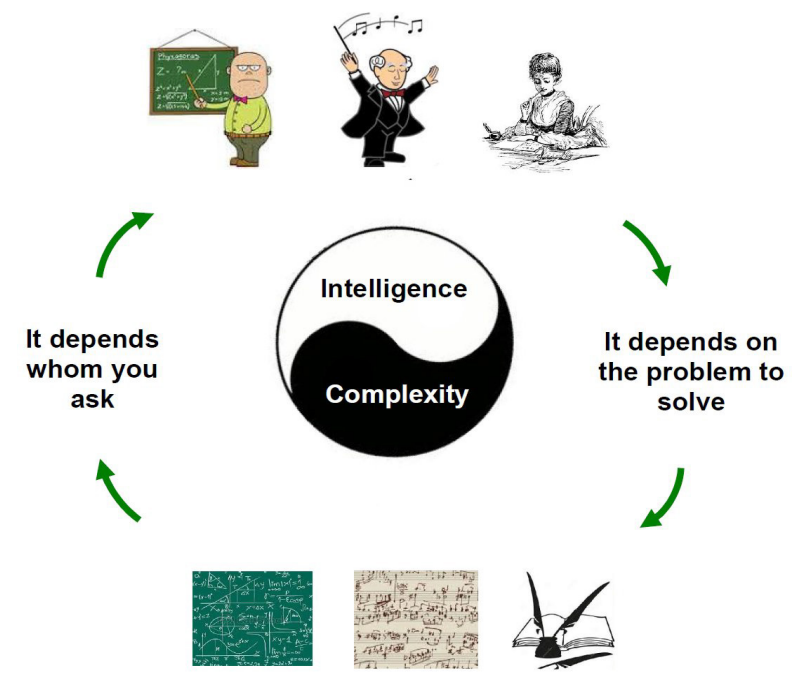

\section{What problem is more complex?}

Figure 2. Intelligence and complexity

The problem solving ability is relativistic in nature, because it is measured in reference to a specific type of problem. For example, who is more intelligent: Mathematician, Composer, or Writer? Obviously, the answer depends on the specific type of problem that is selected for testing: to prove a theorem, compose music, or write a story. By the way, the same relativistic approach could be applied 
to measuring the complexity of the problem by referencing it to a specific intelligent ability. For example, what problem is more complex; to prove a theorem, compose music, or write a story? The answer depends whom we ask: Mathematician, Composer, or Writer (see Fig. 2).

This way, intelligence and complexity are both relativistic in nature and complementary to each other that could be expressed as follows:

$$
\text { Complexity }+ \text { Intelligence }=1
$$

Incidentally, the proposed relativistic approach to complexity is at odds with dominating views. Since its introduction almost 100 years ago, scientists continue define complexity as an absolute category [27], but they still cannot come to terms with the illusive nature of complexity.

However, let us return to the main subject of this chapter the theory of intelligence, which we may call the Relativistic Theory of Intelligence. This theory allows us to measure and compare the intelligent abilities of any systems; living, nonliving, natural or artificial. For example, let us compare the problem-solving ability of a human brain to that of a calculator. If a problem entails a calculation of large numbers, the simple calculator would have a higher score for this specific intelligent ability than a human. However, if we replace the problem with one that requires a common sense, a human will demonstrate his/her superiority. Following this approach any system could be superior in solving a specific problem or performing a specific task. For example, for a molecule of protein, this task is a protein folding; for an enzyme molecule, this task is catalysis of biochemical reactions; for atoms this task is self-assembling into molecules. We humans are overflowing with all sorts of superiorities, but still cannot compete with atoms in assembling even the simplest molecule due to the difficulty of an analytical solution to the n-body problem, so an approximation is used instead.

Measuring the specific intelligent ability by using a specific problem is a straightforward procedure. To compare different intelligences as a collection of various problem-solving abilities, we have to assign a weight factor to each of these abilities. A judgment of intelligence in every society is based on subjective weight factor. In a modern society, this weight factor gave preference to academic abilities. For example, we regard Bob, who speaks Latin and holds a $\mathrm{PhD}$ in philosophy, as more intelligent than Steve, who is a high school dropout. However, Steve has exceptional hunting abilities and if judged by the members of a primitive hunter-gatherer tribe, Steve would have a higher score than Bob despite his impressive Curriculum Vitae.

If someone would mention that "Mr. Smith is very intelligent man," you may want to clarify "Intelligent, to do what?"

\section{From Intelligent Elements to Intelligent Systems}

One of the arguments against panpsychism is that it cannot account for the relation between higher- and lower-order minds; if mind is supposed to exist in atoms, then higher-order minds, such as the ones that humans have, must be some kind of combination or sum of these lesser minds. This argument is called the Combination Problem [7]. An attempt to address this challenge is presented in this chapter.

As we defined in the previous chapter, intelligence is the ability to solve a problem. Obviously, the more complex a problem is, the higher level of intelligence is required to solve this problem. It is apparent that a group of individuals is able to solve a more complex problem than an individual member. Probably, the most efficient way of solving complex problem by a group is by dividing the problem into multiple sub-problems of lower complexity and solving them piece by piece. However, often a problem cannot be divided into simpler sub-problems; in this case, a consensus approach can be used instead. In this respect, each member of the group solves a problem independently and then compares the results with other members; non-identical results will be canceled out as incorrect, but the result selected by the majority represents the solution defined by a group.

Not every body would agree with the consensus as the solution of a problem. They would argue that the majority advocating a particular solution might have little or nothing to do with finding the right answer, in other words, the truth.

The word truth, in the common language, means to be in agreement with fact or reality. However, this word has no single definition about which professional philosophers and scholars agree. According to the Consensus theory [28] truth is whatever is agreed upon by some specified group. According to the theory of Social Constructivism [29] the truth is constructed by social processes and it is historically and culturally specific. Both of these theories implicate that there is no absolute truth, but relative truth only.

Let us go back to the group that is solving a problem using the consensus approach. Say a group of $\boldsymbol{m}$ individuals is solving an open-ended problem that has one correct solution and an infinite number of possible incorrect results. These individuals are working independently, but at the end compare their results. We consider that the group solves this problem (achieves consensus) if at least two members of the group come up with the same answer, while the rest will come with dissimilar results. The probability that at least two members of a group come up with the same answer (solving the problem) is defined as follows:

$$
\mathrm{P}_{\mathrm{g}}=1-\left(1-\mathrm{P}_{\mathrm{m}}\right)^{\mathrm{m}}-\mathrm{m} \mathrm{P}_{\mathrm{m}}\left(1-\mathrm{P}_{\mathrm{m}}\right)^{\wedge(\mathrm{m}-1)}
$$

where:

$\mathrm{P}_{\mathrm{m}}$ the probability of solving a problem by any member of the group.

$\mathrm{P}_{\mathrm{g}}$ the probability that at least two members of the group come up with the same result that meets the definition of consensus solution of the problem. 


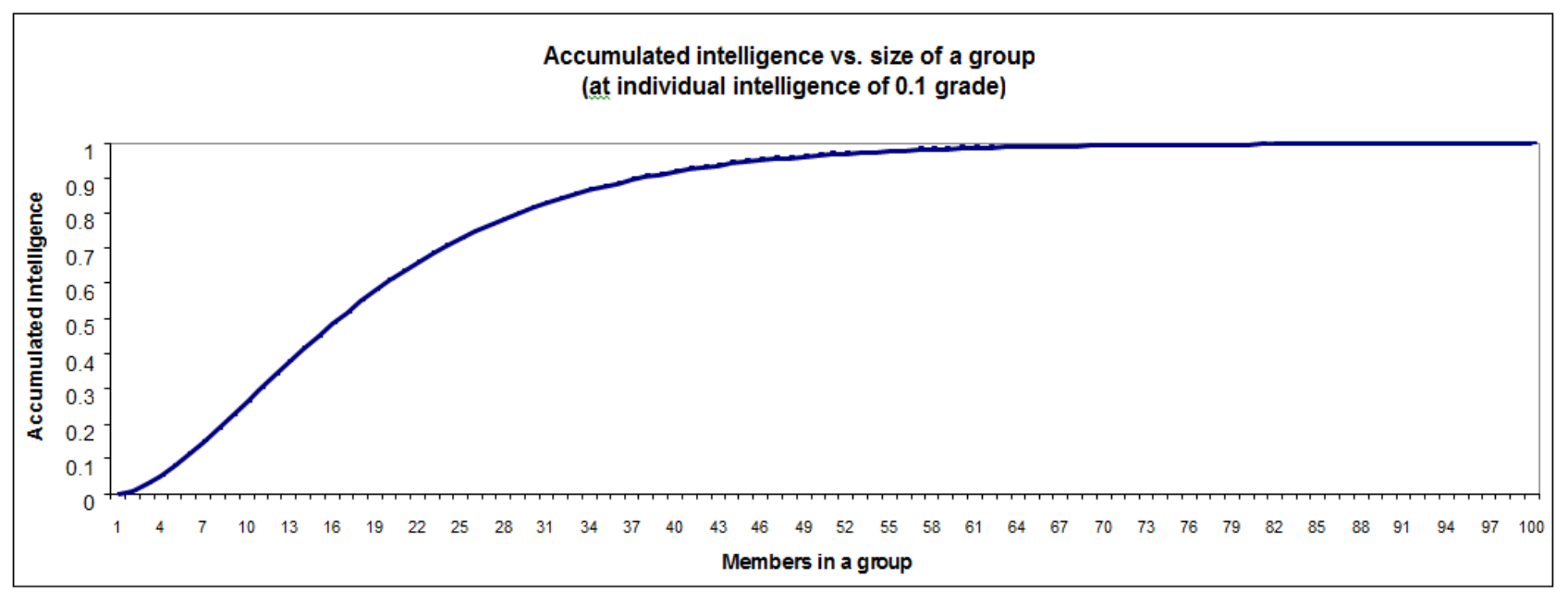

Figure 3. Accumulated intelligence of a group

As seen from Fig. 3, the group accumulates the intelligent abilities of its member, thus having a higher probability of solving a problem.

This way, higher-order intelligence arises from lower-order intelligence as a sum of the lesser intelligences, and in this way a higher mind arises from lower minds.

However, the system may not achieve the needed level of intelligence because its size may be limited. This could be overcome by the formation of multiple systems that cooperate among each other, thus collectively forming a higher level of intelligence. This process of cooperation among intelligent systems is seen here as the foundation of hierarchical organization of matter and intelligence. The hierarchical organization allows unlimited accumulation of intelligence from one hierarchical level to the next, from the limited intelligent abilities of elementary particles to the enormous collective intelligence of human society.

\section{Perception of Intelligence}

By promoting the idea that intelligence is all around us, we should answer these questions: why do not we recognize its presence immediately and directly in any object we touch or observe? Could there be something wrong with our perception of reality?

Indeed, our perception of intelligence is limited only to the mental processes that can be revealed to us directly, mostly in the form of human language. Therefore, we do not recognize the intelligence of other species and even some human mental abilities unless they are rationally communicated. For example, a professional basketball player is making a split second decision based on subconscious mental processes that involve analyzing the arrangement and speed of other players, foreseeing their next move, manipulating the attention of rival players, and only then he or she executes the maneuver in order to score a point. Regardless of the exceptional performance of these mental processes occurring in the athlete's brain, these processes are not observable and therefore cannot be perceived as intelligent. At the same time, if someone provides a rational description of these mental processes, the description itself, regardless of its correctness, will be considered as intelligent. This is why all our abilities that result from non-observable subconscious mental processes are discriminated against from being recognized as truly intelligent. Even such human endeavors as art are not perceived as truly intelligent, but rather genius, gifted, exceptional, talented, etc., because the subconscious mental processes artists employ cannot be traced or rationalized. To demonstrate intelligence, it is not imperative what you accomplished, but how you communicate your achievement. Often well-articulated failure is received as more intelligent than poorly presented success.

Hence, if non-communicable mental processes are not recognizable as intelligent, then nonliving systems have no chance of being perceived as intelligent, regardless of their superiority in performing complex tasks. Elementary particles effortlessly and unmistakably assemble themselves into atoms, while we cannot accurately model them due to the n-bodies problem. Protein molecules perform an exceptionally complex folding process, yet scientists still do not understand this mechanism. Crystallized water forms an abundant variety of near-perfect snowflakes, yet we still not fully understand the mechanism of their creation.

We may perceive intelligence if we are able to observe its problem solving ability. For example, if we observe a man who is building a house, we are witnessing his goal-directed problem solving ability. If this man achieved his goal and built a perfect house, we may never observe this ability again. However, if the house didn't perfectly accommodate all of the needs of this man, he will continue working on rebuilding, improving, expanding, and fixing his house, and this way making his problem solving ability constantly observable.

We are able to observe the goal-directed ability of living systems because they never fully achieve their goal and therefore are constantly adapting. In contrast, nonliving systems always unmistakably achieve their goal of minimum of free energy in their spontaneously occurring dynamics. 
Their problem solving abilities in search for equilibrium always have a $100 \%$ success rate. An electron always finds its way to a positive charge; a chemical reaction always yields the predicted final product; a thrown stone always finds its way to the ground, a river always find its way to the ocean. In contrary, there is no living system that achieves a $100 \%$ success rate in solving their problems; mistakes are inseparable characteristics of living systems. Using this observation, we may draw the line between living and nonliving systems as follows (Warning: Please buckle up we are going upside down):

\section{If a system is solving a problem with a $100 \%$ success rate, it belongs to nonliving systems, however if a system makes mistakes and has success rate $<100 \%$, it belongs to living systems.}

Why living systems are "less intelligent" than non-living systems is a very good question, but to find the answer we need to move to the next chapter.

\section{Connecting the Dots: The Goal, Result, and Origin of Life}

Since we are talking about intelligent systems that solve problems, we have to consider two possible outcomes: the correct answer and an incorrect one. It is natural to assume that if a system is solving a problem, say searching for equilibrium, and gets the correct answer, the system will approach the equilibrium, but the incorrect answer leads it away from its destination.

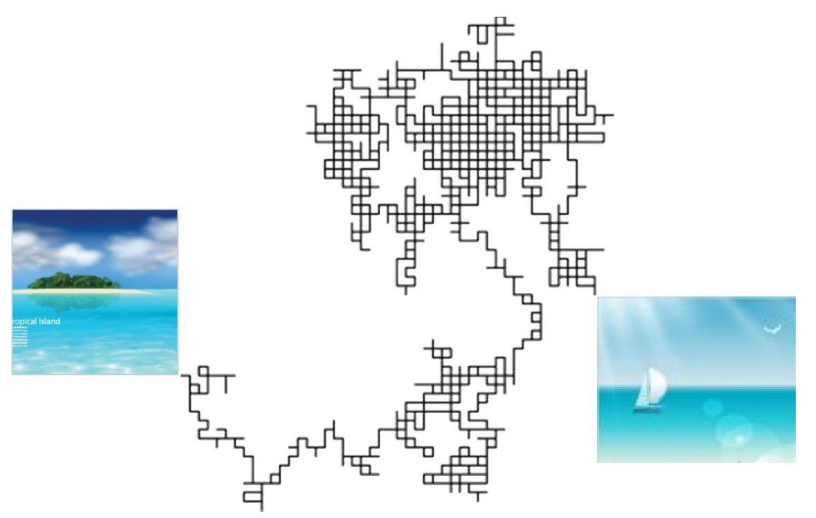

Figure 4. Trajectory of the random search

Imagine a fisherman who lives on a small, remote island. Every day, he takes a boat to fish within a visible distance from the island and therefore he has no problem to find his way back to shore. However, one day, by accident, he gets farther from the island so he cannot see it any more. In this case, the complexity of the problem to find the island may exceed the threshold of this man's ability and as a result the more this man tries to find his island by searching randomly, the further he moves away from it (see Fig. 4). If one observes this fishermen moving away from the island, the observer may assume that the man has the goal to sail towards open water rather than find the island.

This example was used somewhere as an analogy for the emergence of non-equilibrium systems. Non-equilibrium thermodynamics that studies these phenomena considers the two outcomes of the thermo dynamical evolution. If a system is not far from equilibrium, it develops toward equilibrium (as all nonliving systems do), but if a system, as a result of fluctuation, went far away from thermodynamic equilibrium and passes the critical point (Edge of Chaos), the system may engage in chaotic behavior causing various types of self-organization processes that push it farther and farther away from equilibrium. Significant accomplishments have been made to extend these self-organization processes into the realm of living organisms and social systems $[19,30]$.

The analogy between the man, who is moving away from his destination after being lost in the open water, and the processes of self-organization that move away from equilibrium, lead me to think that both of these events manifest the same phenomenon of an intelligent system that is moving away from the destination as a result of being lost.

The evolution of nature on our planet had been developing away from equilibrium toward increasing complexity, but now I would question if this evolution is the "inspiration" of nature. Instead, I would consider that due to random fluctuation, intelligent systems could be pushed far from equilibrium, where the complexity of the problem to find equilibrium exceeds systems' intelligent ability. That leads to inevitable mistakes and instead of approaching equilibrium and simplicity, the system moves further and further from the goal into a web of chaotic and complex behavior. Following this observation, we are coming to the central concept of this paper, which could be stipulated as follows:

Intelligence is the fundamental property of
matter that is not recognizable in nonliving,
equilibrium systems. However, if a system
steers far enough from equilibrium and
passes the critical point, the inevitable
mistakes in search for equilibrium push the
development of this system further away
from its goal in the direction of
self-organization and complexity, revealing
system's intelligent abilities. However, we
do not recognize this property as intelligence;
instead, we call it LIFE.

\section{Abiogenessis and Biological Evolution}

It is often argued that one of the most fundamental laws of nature, the second law of thermodynamics (increasing entropy and disorder), is incompatible with the transformation of matter from disordered atoms and molecules into the self-organization of complex structures 
[12]. However, far from equilibrium, entropy production "takes a back seat," the principle of self-organization dominates and the question of the origin of life appears in a different perspective; once the conditions for self-organization were satisfied, life became as inevitable as rain on a cloudy day [39].

The advent of non-equilibrium systems is the inevitable outcome of the evolution of inanimate matter. This evolution, from simple to increasingly complex molecular systems, leads to exponentially increasing complexity (combinational complexity) to find equilibrium, while the problem solving ability of these systems grows only logarithmically (see Figure 3). Eventually, the complexity of the problem to find equilibrium should exceed the intelligent ability of these systems leading to inevitable mistakes, complexity, and self-organization far from equilibrium. That is seen here as the foundation of Abiogenessis.

In accordance with dominant paradigms, the emergence of life relies on rare (and many argue improbable) chance. According to Neo-Darwinism synthesis, evolution is a blend of a chance and selection; chance in the creation of new genetic variations by mutation, and selection as it favors the propagation of some variations over others. These days, very few scientists argue against biological evolution or the role of natural selection, but there are many who do not agree that random mutations are "creative enough" to provide material for this selection.

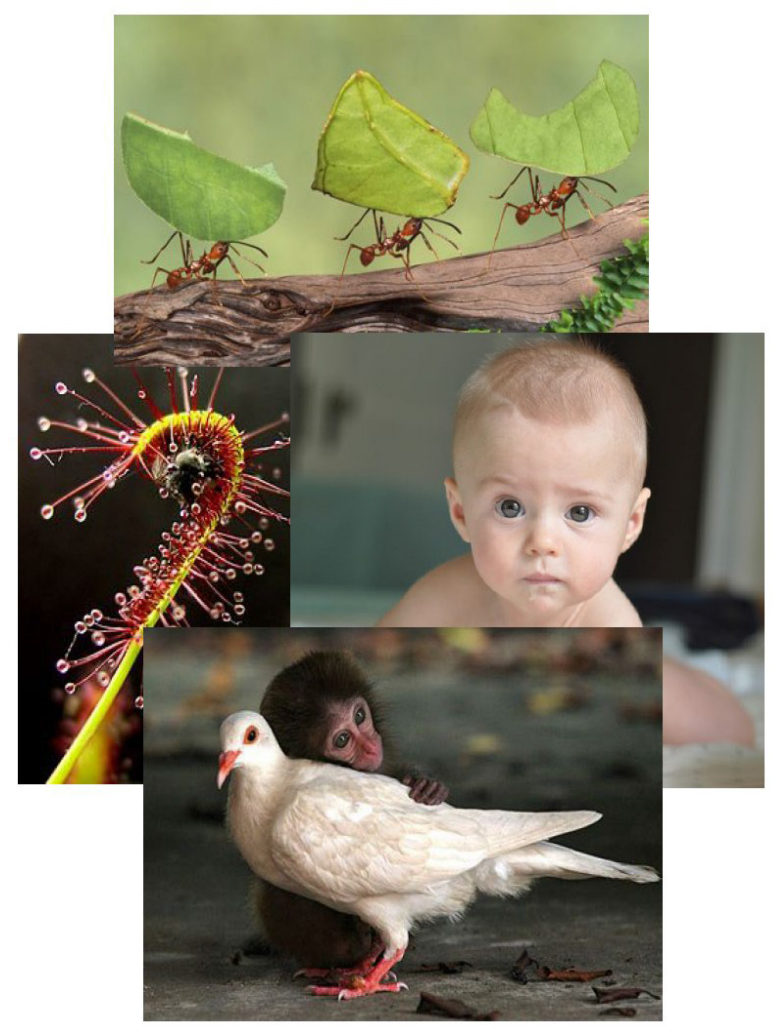

Figure 5. Variety of Minds

The alternative to the mechanism of random mutations is the theory of Intelligent Design that views life as a creation by external intelligence. The attempts to prove this theory are often outside of acceptable scientific methods and most of the time their proponents just point to the gaps of Neo-Darwinism.

The theory of evolution proposed here marries the Darwinian theory of evolution with the theory of Intelligent Design. However, this is a tough marriage and requires concession from both sides; the Darwinian theory of evolution needs to divorce from the mechanism of random mutation and the theory of Intelligent Design needs to divorce from the external intelligence (God or god-like entity) and accept internal intelligence instead. The new theory offers a new road map to biological evolution in which chance and random mutations are replaced with goal-directed intelligent processes. The minds they created are the living testimony of how intelligent these processes are.

\section{The Price of Achieving a Goal}

If life indeed emerged due to the inevitable mistakes in finding equilibrium, the solution it is looking for is death, and living organisms search their entire life to find this answer.

I did not warn the reader to buckle up before presenting this deduction, because no safety belt would help in the collision of good common sense with this disturbing inference about death as a life's goal. Viewing death as goal is in sharp contradiction with the dominant concern of any living organism to preserve its life at any cost.

Regardless of how shocking and counterintuitive the reconciliation of life and death is, we should not be surprised that living systems could be subjected to internal conflicting forces.

Let us look into a familiar living system - human society. One of the most basic conflicts we probably all have experienced is between our obligations to society and our individual needs. People gather into social systems not because they like to be reminded of their obligations, but in order to reach their individual goals of protection and prosperity through exchange and association with others. The tremendous bounty of such cooperation makes social interaction the greatest tool for achieving individual goals. However, if one day all members of society would achieve their individual goals (say, to win the lottery and have a good time) there will be no need for such cooperation. As a result, this society, and consecutively its members, would cease to exist, because they would not survive by themselves. Unfortunately, the association between achieving a goal and a fatal end is counterintuitive, so we may not recognize the warning signs of the approaching fall. Well, life is inherently associated with mistakes.

To move from human societies to individual organisms I should remind the reader that according to the views advocated here, any self-organized system is a social system of conscious intelligent constituents. Therefore, an organism, 
like us, is a social system of intelligent cells.

One of the main characteristics of dividing cells is that their survival depends on replication; inhibition of mitotic cell division leads to their death [41]. Therefore, we should consider the cell division of replicating cells as their vital need. However, unlimited cell replication is in conflict with the carrying capacity of organism. If this conflict, let us call it overpopulation, is not addressed, the destruction of the organism is inevitable. Therefore, for an organism to survive it needs to eliminate the surplus of propagating cells.

The indication of violent resolution and the severity of this conflict is the widespread cell destruction occurring at all stages of an organism's development, all over the animal kingdom as well as in various tissues. For example, the cell death during development of the nervous system may exceed $50 \%$ and death of immune cells prior to full maturation exceeds $95 \%[40,43]$.

The explanation of cell death proposed here, as a product of violent conflicts, contradicts the current paradigm that views cells as programmed to commit suicide at a predetermined period. This programmed cell death, called apoptosis, is triggered by external chemical signals that initiate intracellular (allegedly) "suicidal" metabolic pathways [45].

Presenting cell death as "suicide," regardless that it is triggered by external factors, has the same justification as poisoning a person and calling this a suicide, because it could be presented as caused by the suicidal metabolic pathways built into victim's bodies that were just triggered by poison. The claims about cell suicide are also in drastic contradiction with the observation that cells promote survival at all costs by employing multiple strategies to prevent their death by apoptosis [46]. However, we still stubbornly call it suicide.

It would be natural to expect that the conflict due to overpopulation is in its peek at the time when a growing organism reaches its critical size causing a rising surplus of unwanted dividing cells. This stage of development coincides with the reproductive maturity of organism that exhibits the most common examples of "programmed" cell death. Apoptosis has been more intensively investigated in the reproductive organs than in almost any other system [50]. The link between reproduction, an excess of replicating cells and "programmed" cell death deserves separate consideration, but for now let me just propose that the reproductive life of an organism is an indication of ongoing conflict caused by a surplus of propagating cells; this conflict is in its peek at the onset of reproductive maturity and is resolved at the end of the reproductive life.

Overtime the reproductive activity of an organism gradually decreases and eventually dies out, that should be an indication of achieving the balance between available and required replicating cells. We can say that this is a time of successful resolution of the overpopulation conflict.

However, it may not be a time to celebrate because decreasing the number of propagating cells leads to the aging of the organism's cell population, organism gradual deterioration and eventually to its death. This is the price an organism pays for achieving its goal.

The question needs to be asked: why does the organism, for which "survival at all costs" (including the killing of its own cells) is the ultimate goal, cannot solve the aging problem by simply replacing aging cells with younger ones? The problem is that long-living aging cells, like brain cells, osteocytes, cardiomyocytes, etc., that have long history of cooperation and sharing their conscience experience are the founding members of the organism's collective conscience and therefore their survival is "preserved at all costs." The younger dividing cells come and go, and therefore they are not a significant part of the organism's social hierarchy. Therefore, the aging cells that form collective conscience should be preserved from replacement. Obviously, this is a losing strategy against the entropy driven forces. Well, again, life is inherently associated with mistakes.

While discussing aging and death of an organism we cannot avoid discussing aging and death on the cellular level. Because I consider a cell to be a social system, we should expect that the social conflict of overpopulation also happens inside cells that achieve their critical size. However, the resolution of this conflict seems less violent than in an organism because it is being resolved by separating conflicting "parties" during cell division. However, if the cell stops dividing it should start accumulating aging macromolecules. These molecules are affected by entropy-driven degrading structural changes. For example, entropy driven processes cause gradual protein misfolding leading to weakening or loss of functionality. Cells have some means to replace the malfunctioning protein, but this ability is limited, and with age, the balance between the synthesis and degradation leads to waste acumulation of aged proteins [31,32].

While we consider the aged misfolded macromolecules as waste that need to be discarded, these aged molecules may not view themselves as waste. Some of them, especially those located within nucleus, are part of the cell's collective conscience and therefore they are the subject of "preservation at all costs." These macromolecules after a long and successful career, during which they were being shaped into a somewhat uncomfortable three dimensional work robe, are finally loosened up and ready to "retire" at thermodynamic equilibrium (speaking biologically, death), but maybe still could serve in an advisory role. Unfortunately, when the critical number of such "retired" macromolecules is accumulated, the viability of the cell is compromised, eventually leading to its death. However, this is not what the "retired" macromolecules "expect." Instead, they just want to be as close as possible to the equilibrium state while "enjoying the benefits" of the collective consciousness of the living cell. Unfortunately, following the death of the cell, these macromolecules also undergo breakdown. It may "take them by surprise" because they probably did not recognize the warning signs of the approaching forces of the second law of thermodynamics. Well, life, as we already know, is inherently associated with mistakes. 
There is a striking similarity between the views on death discussed here, as a goal directed process, and the concept of the "death instinct" developed along a very different line of thought by Sigmund Freud. Seeking a primal cause for the repetition instinct, Freud regressed his analysis back to simple multicellular organisms, unicellular organisms, and ultimately to inorganic matter [33]:

“... While speculating on the origin of life and of biological parallels, I concluded that, beside the instinct preserving the organic substance and binding it into ever larger units, there must exist an antithesis, which would seek to dissolve these units and reinstate their antecedent inorganic state; that is, a death instinct..."

This concept was presented in greater detail in Freud's other book, Beyond the pleasure principle, [34] where Freud concluded that "The goal of all life is death":

\section{"... It must rather be an ancient starting point, which the living being left long ago, and to which it harks back again by all the circuitous path of development. If we may assume as an experience admitting of no exception that everything living dies from causes within itself, and return to the inorganic, we can say 'The goal of all life is death', and casting back, 'The inanimate was there before animate."}

The desire for death phenomenon was studied by another great scientist, Elie Metschnikoff [35], who lived around the same time as Sigmund Freud. Examining centenarians, Metschnikoff collected evidences of a desire for death and based on these and other evidences he brought forward the theory that if one attained to the normal span of life, (he estimated it at about 100 years) the desire to live would slowly disappear and be replaced by a desire for death - "a desire to return whence one had come."

In contemporary psychology, the desire of death is a clinical condition. It is known that very elderly may have developed a 'desire for death' related to their frailty, and the fact that their contemporaries have all gone. Loss of close relatives or friends often causes depression, and is the leading factor associated with the desire for death. This is controversial area of scientific inquiry and cannot be taken lightly. However, it may be worth to mention that the association between the desire for death and degenerative processes in the brain [36-38] is in line with views that loss of prominent members of collective conscience, as brain cells are, weaken organism's will to protect itself against the destructive forces of internal socials conflicts. Under these circumstances, giving up the fight and accepting death as a relief could be a desirable outcome.

The discussion presented in this chapter does not pretend to give sufficient explanation for the death phenomenon, but just offers new perspectives that suggest that life and death are deeply interconnected, and we may never fully understand life without understanding death.

\section{Spiritual Knowledge}

Let us return to the example with the fisherman who is lost in the ocean. If given enough time, assuming a limited size of open water and assuming he would survive the long journey, he may eventually find his home and finally be able to rest. However, for a distant observer who may perceive the fisherman's journey as adventures, the final episode could be seen in a different light. For this observer, the transition from what appears to be vibrant and adventurous behavior during the journey, to the quiet life at home, is perceived as the end of dynamic and meaningful life, in other words, death.

This interpretation of events is in line with the dominant scientific views on death, as the termination of biological processes - the only indication of life and the only meaningful form of existence. Contrary to science, spiritual teachings consider death as a transition from life to a different and often more desirable, eternal form of existence. The history of spiritual traditions is thousands of years old and represents a significant portion of human intellectual and cultural knowledge. However, there is plenty of skepticism about the value of this inheritance. I am one of those skeptics, but at the same time, spiritual knowledge should not be entirely discarded by association with religion dogmas. Instead, any area of human knowledge could be viewed as a reflection of the multidimensional reality we are living in. Each of these reflections portrays reality at a different angle and therefore is partial and often distorted. However, the main problem is not in the partiality of this knowledge, but in the ignorance and refusal to recognize this partiality.

The two most powerful institutions, religion and science, have conflicting positions in representing reality. At the same time, there are a number of outstanding scientists and theologians, in the past and present, who do not see a conflict between scientific and spiritual knowledge, so there should be a way to reconciliation. In this regard, panpsychism opens the new opportunities in connecting the tangible physical reality with subtle underlying mental processes. Since mind, according to panpsychism, governs all mental and physical processes, panpsychism could be a bridge between science and spirituality.

One of the main themes presented in various forms in different spiritual teachings is the concept of reincarnation, which is the metaphysical belief that some essential part of human survives physical death to be reborn in a new body. Traditional science rejects reincarnation on the grounds of the absence of a physical process by which a personality could survive death and travel to another body. However, within panpsychism, the link between concept of reincarnation and life-death cycle become feasible. After death the conscious experience of the living organism does not vanish, but is inherited by its disintegrated elements. After being "recycled" to another living organism, those 
elements could bring their previous conscious experience that could be integrated into the collective consciousness of the host. This way, the phenomenon of reincarnation is expected to be universal to any living systems and not exclusive to humans.

The concept of reincarnation could be also mirrored and applied to non-living matter. After death, decomposed materials are returned to a non-organic state, however, they are not the same atoms and molecules they were before. Even though they may be indistinguishable chemically from their previous forms, they are different because they bring the part of the collective conscious experience from living organisms back to non-organic matter. This way, matter becomes more intelligent via a full cycle of which death is an integral part.

\section{Conclusions}

The explanation of the phenomenon of life proposed here calls to replace physicalism with a new paradigm that recognizes mental capacity as a fundamental property of matter. In this paper, I can argue that the new paradigm is superior to physicalism in explaining not only Life and Mind but also physical phenomena, including the notoriously controversial interpretation of quantum mechanics.

There is a high price for accepting this view, because it requires the rejection of the most fundamental scientific paradigms affecting almost every area of knowledge. However, the reward for the transition to the new views justifies the cost, because it promises to eliminate the "weirdness" from quantum mechanics, unite physics with the life sciences, and bridge science with spiritual experience.

The new paradigm leads to a new explanation of the evolution of matter from a disordered state to the emergence of living systems. According to this explanation, systems are continuously in search of equilibrium. If the complexity of this search exceeds the intelligent ability of the system it starts making mistakes, search randomly and gets lost in an ocean of possible solutions, moving further away from equilibrium toward complexity, self-organizing on the way to biological evolution.

It is very important to understand this phenomenon because it affects the development of any intelligent system including our daily life. Every decision we make brings us closer to our goal or leads us away from our destination, if the complexity of the problem exceeds the threshold of our ability. Staying away from this threshold should keep us away from the turbulent forces of inevitable mistakes that would push us further and further from the goal.

The title "The Simplicity of Complex Systems" reflects the proposed relativistic view on complexity. According to this view, the complexity of living systems is a subjective phenomenon and just reflects the current state of our knowledge. Eventually when life will be explained it should lose its status of complexity the same way complex dynamics in the physical world was eventually reduced to simple Newtonian mechanics. It took a couple thousand years to figure it out; that indicates that in the past it was not a simple problem.

Now life and mind phenomena are the most challenging questions science faces. If searching randomly or using the wrong methodology, it may take science another thousand years wandering in the ocean of endless possible solutions, leading us further from the answer. Therefore, another aim of this paper is to question if life sciences are searching in the right direction, or if they are lost and need to reexamine their roots, first and foremost the "mindless" physicalism which regardless of countless attempts has not proven to be a firm fundament in explanation of life. Instead, in the spirit of Schrödinger's quest for new physics for life [54], a new physical theory may be needed to pave the way to understanding Life, Mind, and Death phenomena.

\section{REFERENCES}

[1] Skrbina, D. [2005]: Panpsychism in the West. (Cambridge, MA: MIT Press).

[2] Skrbina, David [2007]: 'Panpsychism,' The Internet Encyclopedia of Philosophy. Retrieved 01/10/2010, from: www.utm.edu/research/iep/p/panpsych.htm

[3] Whitehead, A. [1938]: Modes of Thought. (New York : Macmillan).

[4] Bohm, David [1986). 'A New Theory of the Relationship of Mind and Matter.' Journal of the American Society for Psychical Research 80 (2 \& 3):113-35.

[5] Wright, Sewall [1977]: Panpsychism and science. In John B. Cobb and David Ray Griffin (eds.), Mind in Nature (University Press of America).

[6] Bohm, David [1986). 'A New Theory of the Relationship of Mind and Matter.' Journal of the American Society for Psychical Research 80 (2 \& 3):113-35.

[7] Seager, W. [1995]: 'Consciousness, information and panpsychism,' Journal of Consciousness Studies, 2(3), pp. 272-288.

[8] Griffin, D. R. [1998). Unsnarling the World Knot. (Berkeley, CA : University of California Press).

[9] Birch, C. [2001). 'Why I Became a Panexperientialist.' Paper given at the Australian Association for Process Thought 2001 Conference, 29 November - 1 December 2001, at Latrobe University, Melbourne.

[10] Jammer, Max. [1998). Concept of Forces, (Toronto, Ontario, Canada : General Publishing Company), (see Preface).

[11] Ball, Philip, [2003) 'The Physical Modelling of Human Social Systems,' Complexus; 1:190-206.

[12] Huxley, Julien, [1953]: Evolution in Action (New American Library, N.Y.) p. 11-12.

[13] Levine, Herbert and Rappel, Wouter-Jan. [2000]: 'Self-organization in systems of self-propelled particles,' 
Physical Review E, Vol. 63, No. 1, 017101.

[14] Rummel, R. J. [1976]: Understanding Conflict and War, (Publisher: John Wiley and Sons Inc.).

[15] Peat, F. David [1987]: Interview with David Bohm provided and conducted by F. David Peat along with John Briggs, first issued in Omni magazine, January.

[16] Wolfram, Stephen, [2002]: A New Kind of Science. (Champaign, IL : Wolfram Media).

[17] Daintith, J. [2004]: Oxford Dictionary of Chemistry. (New York : Oxford University Press).

[18] Campbell, Neil A. and Reece, Jane B. [2002]: Biology, $6^{\text {th }}$ edition. (San Francisco, Calif.; London : Addison-Wesley).

[19] Prigogine, Ilya and Stengers, Isabelle [1988]: Order Out of Chaos: Man's new dialogue with nature, (New York : Bantam Books).

[20] Whitley, M. J. and Lee, A. L. [2009) 'Frameworks for Understanding Long-Range Intra-Protein Communication'. In: Curr Protein Pept Sci 10(2): 116-127(12).

[21] Braitenberg, V and Schüz, A [1991]: Anatomy of the Cortex: Statistics and Geometry (NY : Springer-Verlag).

[22] Calvin, William H. [1996) The Cerebral Code: Thinking A Thought In The Mosaics Of The Mind. (Cambridge, MA : MIT Press).

[23] Eccles, John C. [1992]: 'Evolution of consciousness.' Proc. Natl. Acad. Sci. USA Vol. 89 pp. 7320-7324.

[24] Russell, Peter [2007) The Global Brain: The Awakening Earth in a New Century. (Edinburgh : Floris Books).

[25] Gardner, Howard. [1999) Intelligence Reframed: Multiple Intelligences for the 21st Century. (New York : Basic Books).

[26] Wolfram, Stephen, [2002]: A New Kind of Science. (Champaign, IL : Wolfram Media).

[27] Burgin, M. [1982]: 'Generalized Kolmogorov complexity and duality in theory of computations', Notices of the Russian Academy of Sciences, v.25, No. 3, pp.19-23.

[28] Habermas, Jürgen, [1972]: Knowledge and Human Interests, English translation by Jeremy J. Shapiro, (Boston : Beacon Press).

[29] Burr, Vivien [1995). An Introduction to Social Constructionism. (London: Routledge).

[30] Kauffman, Stuart [1995). At Home in the Universe: The Search for the Laws of Self-Organization and Complexity. (New York : Oxford University Press).

[31] Dobson, C.M. [1999]: 'Protein Misfolding, Evolution and Disease', Trends Biochem. Sci. 24, 329-332.

[32] Harry, R. [2002]: 'Aging Concepts and Controversies,' 4th Edition, (California Moody. Thousand Oaks, CA).

[33] Freud, Sigmund [1930]: Civilization and its Discontents, Standard Edition (Copyright (C) 1961 by James Strachey), p. 77.

[34] Freud, Sigmund [1922]: Beyond the Pleasure Principle. Authorized translation from the second German edition by
C.J.M. Hubbac. (The international Pscycho-Analitical Press, London, Vienna).

[35] Cochrane, L. [2003]: 'Elie Metschnikoff and his theory of an 'instinct de la mort',' International journal of epidemiology, 32 (1): 32-36.

[36] Kissane, D.W. and Kelly B.J. and Aust, N.Z. [2000]: 'Demoralization, depression and desire for death: problems with the Dutch guidelines for euthanasia of the mentally ill,' J Psychiatry. Apr; 34(2):325-33.

[37] Videbech, P [1997). 'MRI findings in patients with affective disorder: a meta-analysis'. Acta Psychiatrica Scandinavica 96 (3): 157-68.

[38] Duma, R. [2004]: 'Depression: a case of neuronal life and death?' Biological Psychiatry. Volume 56, Issue 3, Pages 140-145.

[39] Prigogine, Ilya and Stengers, Isabelle [1988]: Order Out of Chaos: Man's new dialogue with nature, (New York : Bantam Books).

[40] Rathmell, J.C. and Thompson, C.B. [2002). 'Pathways of apoptosis in lymphocyte development, homeostasis, and disease.' Cell 109 (Suppl): S97-107.

[41] H Vakifahmetoglu1, M Olsson1 and B Zhivotovsky. Cell Death and Differentiation (2008 15, 1153-1162; doi:10.1038/cdd.2008.47; published online 11 April 2008.

[42] Solomon M, Belenghi B, Delledonne M, Menachem E, Levine A (1999). "The involvement of cysteine proteases and protease inhibitor genes in the regulation of programmed cell death in plants". The Plant Cell 11 (3): 431-44.).

[43] (Hutchins, J.B., and Barger, S.W. [1998]: 'Why neurons die: cell death in the nervous system.' Anatomical Record, The New Anatomist 253: 79-90.)

[44] Campbell, Neil A. and Reece, Jane B. [2002]: Biology, 6th edition. (San Francisco, Calif.; London : Addison-Wesley).

[45] (Krysko, Dmitri V. [2006]: 'Cell death: initiation, propagation and clearance.' (Department of Anatomy, Embryology, Histology, Medical Physics and Department for Molecular Biomedical Research, Ghent University). Ph. D. thesis in Medical Sciences: p. 7.).

[46] AJ Kole, RP Annis and M Deshmukh Mature neurons: equipped for survival Cell Death and Disease (2013) 4, e689; doi:10.1038/cddis.2013.220; published online 27 June 2013.

[47] Karplus, Martin [1997]: 'The Levinthal paradox: yesterday and today. Folding and Design', 2(4): S69-75

[48] Capra, Fritjof, [1996]: The Web of Life: A New Scientific Understanding of Living Systems (New York : Anchor Books), p. 28.

[49] Laubichler, M.D. and Wagner, G.P. [2001]: 'How Molecular is Molecular Developmental Biology? A reply to Alex Rosenberg's Reductionism redux: computing the embryo', Biology and Philosophy, 16:53-68.

[50] Roger Gosden and Norah Spears. Programmed cell death in the reproductive system British Medical Bulletin 1997, 52 (No. 3) 644-66)

[51] Wick, David. [1995]: The Infamous Boundary: Seven Decades of Heresy in Quantum Physics (New York : Copernicus). 
[52] Timothy O'Connor. Emergent Properties, American Philosophical Quarterly, 31 (1994), 91-104

[54] Schrodinger, Erwin. What is Life? (Cambridge Press, 1967).

[53] Alexander Backlund, (2000) "The definition of system", Kybernetes, Volume 29, Issue 4. pp.444-451 